\title{
Stillen reduziert kindliches Diabetesrisiko unabhängig von Art und Dauer
}

Lund-Blix NA et al. Infant feeding and risk of type 1 diabetes in two large scandinavian birth cohorts. Diabetes Care 2017; 40: 920-927

Nicht genetische Faktoren spielen eine wichtige Rolle bei der Entstehung des Typ-1-Diabetes. Schon vor über 30 Jahren wurde die Hypothese aufgestellt, dass Stillen möglicherweise vor Diabetes schützt. Studien, die diese Hypothese stützen, sind häufig retrospektiv oder prospektiv mit geringer Probandenzahl. Eine neue Studie aus Skandinavien mit zwei großen Schwangerschaftskohorten sollte den Zusammenhang besser beleuchten. 
Für den protektiven Effekt des Stillens auf das Diabetesrisiko kommen zahlreiche biologisch plausible Mechanismen in Frage, wie beispielsweise der Schutz der Muttermilch vor möglicherweise diabetogenen Infektionen. Die Metaanalyse von Beobachtungsstudien zeigt, dass Kinder, die über 3 oder mehr Monate teilgestillt oder mindestens 2 Wochen vollgestillt werden, ein 15-30\% geringeres Risiko für Typ-1Diabetes haben. Da es sich hierbei lediglich um Fall-Kontroll-Studien handelt, sind die Rolle des Stillens und die optimale Stilldauer immer noch nicht genau geklärt. $\mathrm{Ni}$ colai Andre Lund-Blix vom Norwegian Institute of Public Health in Oslo und seine Kollegen untersuchten nun in einer groß angelegten Studie mit mehr als 150000 Kindern, welche Assoziation zwischen Stillen und der Entwicklung eines Typ-1-Diabetes bei Kindern besteht. Tatsächlich schützte Stillen wohl vor der Entwicklung eines Typ-1-Diabetes - interessanterweise schien dieser Schutz unabhängig von der Art und Dauer des Stillens zu sein.

Lund-Blix und Kollegen griffen für die Studie auf zwei der weltgrößten, populationsbasierten Schwangerschaftskohorten zurück, der norwegischen Mother and Child Cohort Study (MoBa) und der dänischen National Birth Cohort (DNBC). Insgesamt lagen Daten von 155392 Kindern vor. In MoBa betrug das durchschnittliche Kindesalter am Ende des Beobachtungszeitraums 10,2 Jahre, in DNBC 14,0 Jahre. In MoBa wurden Informationen zur Ernährung des Kindes über Fragebogen ermittelt, in DNBC über Telefoninterviews. Die Befragungen fanden 6 und 18 Monate nach Geburt statt. Die Studienleiter stellten 2 Definitionen auf: „Vollstillen“ bedeutet, dass Kinder nur Muttermilch erhielten, lediglich Getränke auf Wasserbasis und Vitaminpräparate waren ergänzend erlaubt. Beim „Teilstillen“ erhielten die Säuglinge zusätzlich zur obigen Definition Babymilchpulver, feste oder halbfeste Kost.

In beiden Kohorten zusammen entwickelten insgesamt 504 Kinder einen Typ-1-Diabetes. Das Durchschnittsalter bei Diagnosestellung betrug 6,7 Jahre in MoBa und 8,5 Jahre in DNBC. Die Inzidenz des Typ-1Diabetes pro 100000 Personenjahre war 30,5 in MoBa und 23,5 in DNBC.
In MoBa gaben 0,8\% der Teilnehmerinnen an, dass sie ihr Kind nie gestillt hatten; 13,8\% stillten ihre Kinder 6 oder mehr Monate voll, während 38,5\% mehr als 12 Monate teilstillten. In der DNBC gaben 2,4\% der Teilnehmerinnen an, dass sie ihr Kind nie gestillt hatten; 6,3\% stillten ihre Kinder 6 oder mehr Monate voll, 20,2\% gaben Teilstillen für 12 oder mehr Monate an.

Das Risiko, einen Typ-1-Diabetes zu entwickeln, war für Kinder, die überhaupt nicht gestillt wurden, um das 2-Fache erhöht (HR 2,29 für kein Stillen vs. Teilstillen $\geq 12$ Monate, HR 2,31 für kein Stillen vs. Vollstillen $\geq 6$ Monate).

Bei den Stillkindern war das Risiko, einen Typ-1-Diabetes zu entwickeln, sowohl unabhängig davon, wie lange die Kinder gestillt wurden, als auch davon, ob sie volloder teilgestillt wurden. Des Weiteren fanden die Autoren keinen Zusammenhang zwischen dem Risiko einer Diabetesentwicklung und dem Zeitpunkt, zu welchem die Mütter Babymilchpulver oder feste Kost zusätzlich zur Säuglingsernährung einführten.

Schlossen die Autoren die Daten derjenigen Kinder aus der Analyse aus, deren Eltern selbst an Diabetes litten $(1,1 \%$ in MoBa und $0,3 \%$ in DNBC), kamen sie zu den identischen Ergebnissen, die oben für die Gesamtkohorte beschrieben sind.

FAZIT

Das wichtigste Ergebnis der großangelegten prospektiven Studie ist, dass Kinder, die nicht gestillt werden, ein 2-fach höheres Risiko als Stillkinder haben, an Typ-1-Diabetes zu erkranken. Wichtig scheint dabei lediglich, dass überhaupt gestillt wird: Bei den Stillkindern hing das niedrigere Risiko weder davon ab, ob ein Kind voll- oder teilgestillt wurde, noch davon, ob es nur sehr kurz oder über mehrere Monate gestillt wurde.

Dr. Michaela Bitzer, Tübingen 